\title{
Polarizability of electrically induced magnetic vortex "atoms"
}

\author{
P.I. Karpov ${ }^{1, \star}$ and S.I. Mukhin ${ }^{1}$ \\ ${ }^{1}$ National University of Science and Technology "MISiS", Leninsky avenue 4, 119991, Moscow, Russia
}

\begin{abstract}
Electric field control of magnetic structures, particularly topological defects in magnetoelectric materials, draws a great attention, which has led to experimental success in creation and manipulation of single magnetic defects, such as skyrmions and domain walls. In this work we explore a scenario of electric field creation of another type of topological defects - magnetic vortices and antivortices. Because of interaction of magnetic and electric subsystems each magnetic vortex (antivortex) in magnetoelectric materials possesses quantized magnetic charge, responsible for interaction between vortices, and electric charge that couples them to electric field. This property of magnetic vortices makes possible their creation by electric fields. We show that the electric field, created by a cantilever tip, produces a "magnetic atom" with a localized spot of ordered vortices ("nucleus" of the atom) surrounded by antivortices ("electronic shells"). We analytically find the vortex density distribution profile and temperature dependence of polarizability of this structure and confirm it numerically by Monte Carlo simulation.
\end{abstract}

\section{Introduction}

Electric field control of magnetization and magnetic topological defects is very promising for creation of new types of magnetic memory devices. Magnetoelectric materials, such as type-II multiferroics [1] are particularly appealing for this purpose. The use of domain walls and other topological defects in multiferroics [2] for magnetic storage are very promising since they provide a mechanism of very dense packing of information, while topological nature protects information from loss under the influence of external perturbations such as heating or mechanical action. Among the bright examples of such topological defects are skyrmion lattices [3], as well as individual skyrmions observed in magnetoelectric materials [4], magnetic vortices $[5,6]$, and domain walls $[7,8]$.

In this paper we report our recent results [9]. We consider theoretically a phenomenological model developed by Mostovoy [10] for a type-II multiferroics and apply it to a thin-film material with easy-plane symmetry. We show both analytically and numerically that in this case a strong enough locally concentrated electric field may cause formation of a "magnetic atom" that consists of a vortex "nucleus" surrounded by antivortex "shells". This idea allows us to propose an experimental realization for 2D Coulomb plasma in a trap and make predictions about its electric polarizability.

\section{The model}

In order to describe a layered material with coupled magnetic and electric subsystems we use the following phenomenological model [9]. We write the total free energy

\footnotetext{
^e-mail: karpov.petr@gmail.com
}

density as a sum of parts arising from electric polarization, magnetization, and magnetoelectric coupling [10]:

$$
\begin{array}{r}
w=\frac{P^{2}}{2 \chi_{e}}-\mathbf{E P}-\frac{\mathbf{E}^{2}}{8 \pi}-\gamma \mathbf{P}((\mathbf{M} \nabla) \mathbf{M}-\mathbf{M}(\nabla \mathbf{M}))+ \\
+\frac{\alpha}{2}\left[\left((\partial \mathbf{M} / \partial x)^{2}+(\partial \mathbf{M} / \partial y)^{2}\right] .\right.
\end{array}
$$

We assume that $\mathbf{M}$ is the primary order parameter, which can induce $\mathbf{P}$. Thus we can find polarization by minimizing (1) with respect to $\mathbf{P}$ :

$$
\mathbf{P}=\gamma \chi_{e}((\mathbf{M} \nabla) \mathbf{M}-\mathbf{M}(\nabla \mathbf{M}))+\chi_{e} \mathbf{E} .
$$

Because of the thin-film geometry of the sample, magnetization tends to be parallel to the film plane, otherwise the demagnetizing field energy would greatly increase; also, material can possess an easy-plane type of symmetry. Hence we restrict the magnetization to lie in the plane

$$
\mathbf{M}(\mathbf{r})=M_{0}(\cos \phi(\mathbf{r}), \sin \phi(\mathbf{r}), 0) .
$$

Using the angle $\phi$, the polarization can be written as

$$
\mathbf{P}=\gamma \chi_{e} M_{0}^{2}\left(-\partial_{y} \phi, \partial_{x} \phi, 0\right)+\chi_{e} \mathbf{E} .
$$

Substituting (3) and (4) into (1), we find that the electric, magnetic, and magnetoelectric parts of the energy combine to

$$
\begin{array}{r}
w=\frac{1}{2}\left(\alpha M_{0}^{2}-\chi_{e} \gamma^{2} M_{0}^{4}\right)(\nabla \phi)^{2}- \\
-\chi_{e} \gamma M_{0}^{2}\left(\partial_{x} \phi E_{y}-\partial_{y} \phi E_{x}\right)-\frac{1}{2} \chi_{e} E^{2} .
\end{array}
$$

The expression for energy $W=\int w d V$ is similar to that of the XY model: $W_{X Y}=\frac{1}{2} \rho_{s} \int(\nabla \phi)^{2} d S$ with an effective spin-wave stiffness $\rho_{s}=\left(\alpha M_{0}^{2}-\chi_{e} \gamma^{2} M_{0}^{4}\right) h$, where $h$ 
is the film thickness. The magnetoelectric coupling just renormalizes the spin stiffness of the XY model. For typical values of parameters [9], $\alpha M_{0}^{2}$ is greater than $\chi_{e} \gamma^{2} M_{0}^{4}$ by several orders of magnitude; therefore, $\rho_{s}$ remains almost unchanged by magnetoelectric interaction and we use $\rho_{s} \approx \alpha M_{0}^{2} h$. Despite this, some interesting features appear. It is known that in the XY and related models the magnetic vortices play the crucial role [11]. Vortex placed at the origin has the form $\phi=k \arctan \frac{y}{x}+\phi_{0}$, where $k$ is the winding number of the vortex. Because of magnetoelectric interaction (2), magnetic vortex acquires an electric charge $k q_{e}$ [10], where

$$
q_{e}=2 \pi \gamma \chi_{e} M_{0}^{2} h .
$$

Neglecting small electrostatic interaction of vortices we consider only their magnetic interaction with 2D Coulomb potential $W= \pm 2 q_{m}^{2} \ln (r / a)$ [11], where $a$ is the shortdistance cut-off of order the lattice spacing and $q_{m}$ is the "magnetic charge":

$$
q_{m}^{2}=\pi \rho_{s} \approx \alpha M_{0}^{2} h .
$$

Here and further we put for simplicity vorticities to be $k= \pm 1$, since vortices with higher winding numbers are energetically unstable.

The magnetic energy of a neutral system of $N$ vortices and $N$ antivortices with distances $r_{i j}$ between $i$-th and $j$-th (anti-) vortex cores and vorticities $k_{i}= \pm 1$ is [12]

$$
W_{m}=-2 q_{m}^{2} \sum_{i<j}^{2 N} k_{i} k_{j} \ln \frac{r_{i j}}{a} .
$$

\section{Vortex - antivortex pairs creation by electric field}

In this section we explore the basic idea of the paper, that a strong non-uniform electric field can break the "magnetic vacuum" of magnetoelectric material via creation of vortex-antivortex pairs. Fig.1 shows the typical geometry

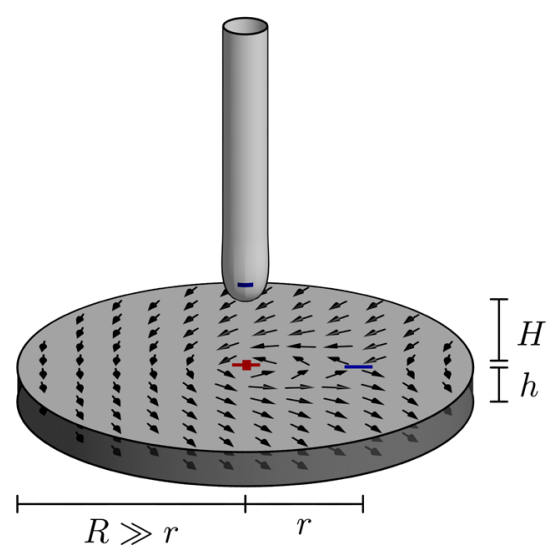

Figure 1. Geometry of the system. Sample is a disk with radius $R$ and thickness $h$. Above its center at the height $H$ the tip of the cantilever with a negative charge $-Q_{t i p}$ is placed.

of the experiment that we keep in mind (which was proposed in Refs. [6, 13]). An electric field is produced by the tip of a cantilever with a fixed point charge $-Q_{t i p}<0$.
Because of the magnetoelectric interaction, strong enough electric field can create magnetic vortices. Here we work in the limit of low temperatures, where there are no thermally activated vortex-antivortex (VA) pairs.

The electric potential produced by the tip is

$$
\varphi(r)=-\frac{Q_{t i p}}{\sqrt{r^{2}+H^{2}}}
$$

where $H$ is the distance from the tip to the sample, and $r$ is the polar radius. We assume that the sample is thin: its thickness $h \ll H$, so we neglect the variation of the electric field in the transverse to the film direction inside the film.

Now we want to find the critical value of the electric field that creates the first vortex-antivortex pair at $T=0$ in a sample of big enough size to accommodate the pair as a whole. Estimates for the case of small samples were made in [13].

Let the electric field produces one VA pair with the vortex situated at the center of the disk and antivortex at the distance $r$ from its center (for big samples $r \ll R$ ). The energy of the pair as a function of $r$ consists of the magnetic energy (8) of the VA interaction and the magnetoelectric part due to electric charge (6) and electrostatic potential (9):

$$
W=2 q_{m}^{2} \ln \frac{r}{a}+Q_{t i p} q_{e}\left(\frac{1}{\sqrt{r^{2}+H^{2}}}-\frac{1}{H}\right) .
$$

Minimizing the energy with respect to the distance between the vortex and the antivortex $r$ we obtain the following optimal distance:

$$
r \approx \frac{Q_{t i p} q_{e}}{2 q_{m}^{2}} .
$$

Note that for in the small sample case $r>R$ the antivortex leaves the sample and only vortex remains (which was analyzed in [13]). Substituting (11) into (10) we find the critical charge $Q_{t i p}^{\text {crit }}$, above which the first VA pair forms:

$$
Q_{\text {tip }}^{\text {crit }}=\frac{2 q_{m}^{2} H}{q_{e}} \ln \frac{H}{a} .
$$

We also define the critical voltage of the tip as voltage produced at the nearest to the tip point of the sample:

$$
\varphi_{0}^{\text {crit }}=\frac{Q_{t i p}^{\text {crit }}}{H}=\frac{2 q_{m}^{2}}{q_{e}} \ln \frac{H}{a}
$$

Higher values of tip voltages can lead to systems with several vortex-antivortex pairs. Estimates for the prototypic type-I multiferroic $\mathrm{BiFeO}_{3}$ give $\varphi_{0}^{\text {crit }} \simeq 20 \mathrm{~V} \times \ln (H / a)$ and for type-II multiferroic TbMnO3: $\varphi_{0}^{\text {crit }} \simeq 0.3 \mathrm{~V} \times \ln (H / a)$ (see [9] for the details).

\section{Vortex distribution in continuous approximation}

In this section we construct an analytical theory for vortex distribution in the continuous approximation. It proves 
that even such a crude approximation captures many effects and allows us to find the vortex distribution profile and the number of vortices for given $Q_{\text {tip }}$.

The number of vortices in the system is not fixed, but is rather determined by the condition of the energy minimum. Here we calculate self-consistently the number of vortices $N$ created by the tip. We work in the continuoum limit [which means that at least $N \gg 1$, so $Q_{t i p} \gg Q_{t i p}^{c r i t}$; see (12)]. Let $n_{v}(\mathbf{r})$ and $n_{a}(\mathbf{r})$ be concentrations of vortices and antivortices, respectively; and $n(\mathbf{r})=n_{v}(\mathbf{r})-n_{a}(\mathbf{r})$.

Consider a ring from $r$ to $r+d r$. In the ground state there is a balance between the electric and magnetic interactions. The magnetic interaction pushes vortices outside while the external electric force pulls them inside the ring (and vice versa for antivortices). From (9) external electric force acting on a given area element with electric charge $q_{e} d N=q_{e} n d S$ is

$$
F_{e l}=q_{e} d N \frac{d \varphi}{d r}=Q_{t i p} q_{e} n d S \frac{r}{\left(r^{2}+H^{2}\right)^{3 / 2}} .
$$

From (8) the magnetic force between two equal charges magnetic $q_{m}$ is $2 q_{m}^{2} / r$. We can now calculate the magnetic force acting at the considered area element $d S$ with magnetic charge $d q_{m}=q_{m} n d S$. Applying Gauss's theorem to magnetic charges we get the magnetic force:

$$
F_{\text {magn }}=\frac{2 d q_{m} q_{m}^{\text {inside }}}{r}
$$

where $q_{m}^{\text {inside }}=q_{m} \int n(\mathbf{r}) d^{2} r=2 \pi q_{m} \int_{0}^{r} n(r) r d r$ is a net magnetic charge inside the ring. Equating (14) and (15) we obtain the vortex distribution density profile:

$$
n(r)=\frac{Q_{t i p} q_{e}}{4 \pi q_{m}^{2}} \frac{2 H^{2}-r^{2}}{\left(r^{2}+H^{2}\right)^{5 / 2}}
$$

Fig.2 shows plot for $r n(r)$, which is proportional to the number of vortices at distance $r$. Vortices are concentrated inside a small circle of radius $r_{0}=H \sqrt{2}$, antivortices are smeared over the sample outside $r_{0}$ with their density decaying as $r n(r) \sim-1 / r^{2}$.

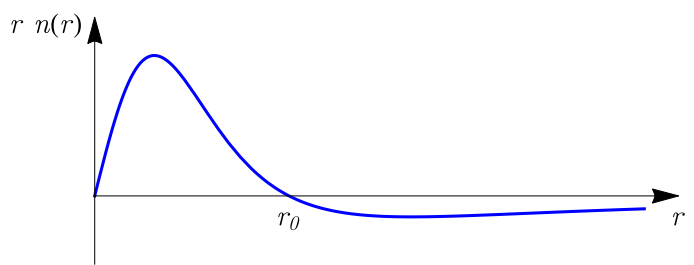

Figure 2. Schematic plot for $r n(r)$ vs $r ; n(r)$ is given by (16).

From (16) we get the total topological charge of vortices and antivortices inside a circle of radius $r$ :

$$
N(r)=2 \pi \int_{0}^{r} n(r) r d r=\frac{Q_{t i p} q_{e}}{2 q_{m}^{2}} \frac{r^{2}}{\left(r^{2}+H^{2}\right)^{3 / 2}}
$$

Now we can calculate the total number of vortices. Border between vortices and antivortices lies at $r_{0}=H \sqrt{2}$, so

$$
N=\frac{1}{3^{3 / 2}} \frac{Q_{t i p} q_{e} / H}{q_{m}^{2}} .
$$

\section{Numerical simulation}

We modeled a system of pointlike vortices and antivortices, which interact between themselves (with the magnetic energy (8)) and with the external electric field created by the cantilever's tip (the electrostatic potential is given by (9)). The system shows a "glassy" behavior; i.e., there are many local minima that are close to the global one. In order to find a state corresponding to such a local energy minimum we performed simulated annealing using the Monte Carlo (MC) method. Three types of MC steps were used: movement of a vortex or an antivortex, creation of the VA pair, and destruction of the VA pair. We cooled the system from high temperatures $T_{\text {start }} \simeq q_{m}^{2}>T_{B K T} \simeq q_{m}^{2} / 2$ (which allowed the system to find the optimal number of vortices) down to as low as $T_{\text {stop }}=10^{-6} q_{m}^{2}$, depending on the numerical experiment.

\subsection{Number of vortices and the critical field}

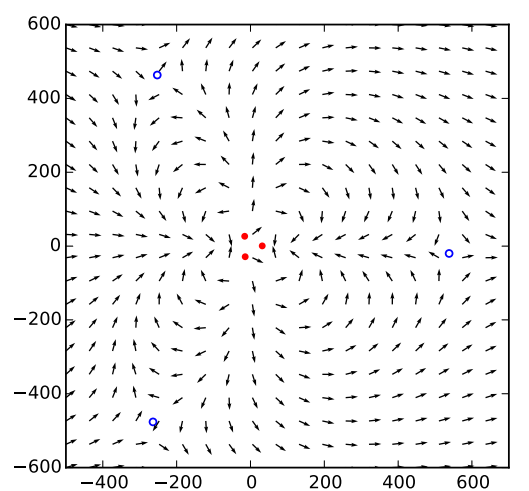

Figure 3. Configuration of a vortex-antivortex system for $Q_{t i p} q_{e} / H=23 q_{m}^{2}$ at $T=0.001 q_{m}^{2}$. Vortex (filled circles) and antivortex cores (open circles) are shown; arrows show magnetization. Coordinates are given in the units of lattice spacing $a$.

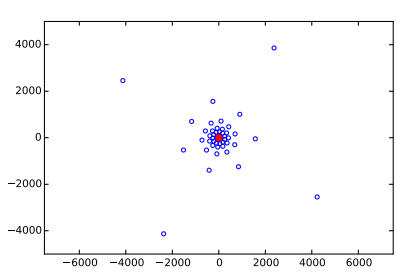

(a)

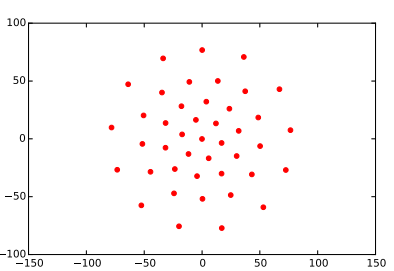

(b)
Figure 4. Configuration of vortex-antivortex system for $Q_{\text {tip }} q_{e} / H=240 q_{m}^{2}$ at $T=10^{-6} q_{m}^{2}$. Vortex (filled circles) and antivortex cores (open circles) are shown. (a) Antivortex subsystem; vortex "nucleus" is not resolved. (b) Vortex subsystem.

As we described in Secs.3 and 4, the charged tip produces magnetic patterns ("magnetic atoms") in the system. Fig.3 shows a typical low-temperature distribution of magnetization in the system for moderate values of tip voltages, for which $N=3$ vortices and 3 antivortices are created.

Fig.4 shows a typical low-temperature configuration of the system for higher values of the tip's voltage. Vortices and antivortices are arranged in a highly regular structure that is reminiscent of the structure of an atom: negatively 


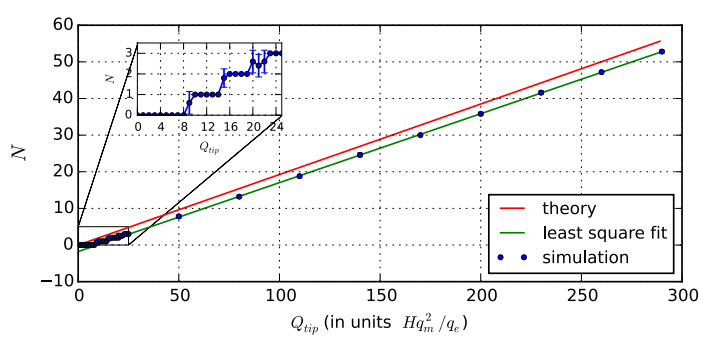

Figure 5. Plot for $N$ vs $Q_{\text {tip }}$. The inset shows zoom of the plot for range $Q_{\text {tip }}=0 \div 24 H q_{m}^{2} / q_{e}$, from which $Q_{\text {tip }}^{\text {crit }} \approx 9 H q_{m}^{2} / q_{e}$.

charged antivortex outer concentric circular "shells" surround the positive vortex "nucleus". Fig.4b depicts only this vortex "nucleus" of the "atom".

Fig.5 shows $N\left(Q_{t i p}\right)$ dependence for the ground states of the system with varying $Q_{t i p}$. The inset shows zoom for small $Q_{\text {tip }}$ : step-like dependence is due to the fact that the number of vortices is always integer. For higher $Q_{\text {tip }}$ the dependence is step-like as well, but the steps are not resolved, and only linear monotonous part of the dependence can be seen. Analytical result (18) and the numerical modeling are in a good agreement.

\subsection{Polarizability of big samples}

Here we numerically find the temperature dependence of the polarizability for big samples. We performed cooling at a fixed value of electric field $E$, and then independently repeated this for different values of $E$. Fig. 6 shows the temperature dependence of the polarizability in log-log coordinates, which in the limit of small $\beta(=1 / T)$ is fitted by $\ln \alpha \approx 0.74 \ln \left(\beta q_{m}^{2}\right)+$ const. This dependence is consistent with our theoretical estimate $\alpha \sim 1 / T^{1-\eta}$, with $\eta \approx 0.26$.

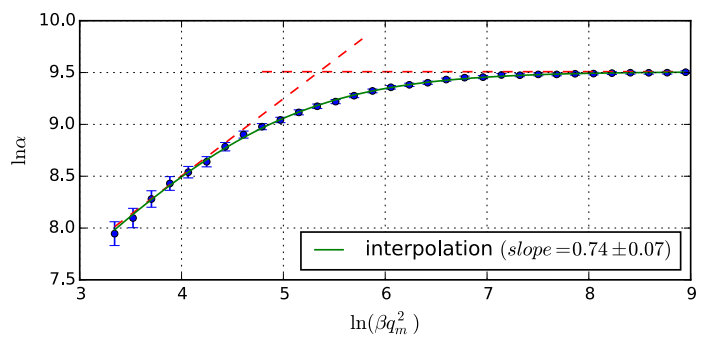

Figure 6. $\ln \alpha$ vs $\ln \left(\beta q_{m}^{2}\right)$ plot (for $Q_{t i p} q_{e} / H=200 q_{m}^{2}, N=$ 36). Symbols show data with their standard deviation; solid line shows fitting. Slope of the dashed inclined line is $0.74 \pm 0.07$.

\section{Conclusions}

Interaction of magnetic and electric subsystems in magnetoelectric materials makes possible the creation of magnetic structures by external electric field. In this paper we have presented analytical and numerical analysis of such quasi-2D materials, where "atom"-like patterns of magnetic vortices and antivortices are produced by a strong electric field, and studied the structure and electric polarizability of these magnetic "atoms".
When the cantilever's electric field exceeds some critical value, the first vortex-antivortex pair forms. Increasing electric field produces more and more vortices and antivortices. We found analytically and numerically the critical voltage required to create the first vortex-antivortex pair, and found the dependence of the number of vortex pairs on the applied voltage. We have shown that a "magnetic atom" forms: a "nucleus" consists of vortices arranged in a lattice-like structure with a short-range order; it is surrounded by antivortices forming outer concentric circular "shells". We obtained the vortex density profile in a continuous approximation.

In the type-II multiferroic-like materials studied here, vortices carry a positive and antivortices a negative electric charge; therefore an additional weak in-plane electric field pulls them in opposite directions, thus creating a polarization. In this work we have studied the temperature dependence of electric polarizability of such "magnetic atoms". By Monte Carlo simulation we deduced that polarizability $\alpha$ behaves at low temperatures like $1 / T^{1-\eta}$, with $\eta \approx 0.26$.

\section{Acknowledgements}

We acknowledge the financial support of the Ministry of Education and Science of the Russian Federation in the framework of Increase Competitiveness Program of NUST MISiS (No. K2-2016-067). Figs.1, 3, 4 are reprinted with permission from P.I. Karpov and S.I. Mukhin, Phys.Rev. $B$ 95, 195136 (2017) doi.org/10.1103/PhysRevB.95.195136. Copyright (2017) by the American Physical Society.

\section{References}

[1] D.I. Khomskii, Physics 2, 20 (2009).

[2] J. Seidel, chapter in "Multiferroic Materials: Properties, Techniques, and Applications", ed. J. Wang (CRC Press, 2016), p.315.

[3] S. Seki et.al., Science 336198 (2012).

[4] P.-J. Hsu et.al., Nat. Nanotechnol. 12, 123 (2017).

[5] K.T. Delaney et.al., Phys.Rev.Lett. 102, 157203 (2009).

[6] A.P. Pyatakov et.al., JMMM. 324, 3551 (2012).

[7] A.S. Logginov et.al., Appl. Phys. Lett 93, 182510 (2008).

[8] A.P. Pyatakov et.al, EPL 93, 17001 (2011).

[9] P.I. Karpov, S.I. Mukhin, Phys. Rev. B 95, 195136 (2017).

[10] M.V. Mostovoy, Ferroelectricity in spiral magnets. Phys.Rev.Lett. 96, 067601 (2006).

[11] J.M. Kosterlitz and D.J. Thouless, J.Phys.C 6, 1181 (1973).

[12] P.M. Chaikin and T.C. Lubensky, Principles of condensed matter physics, CUP, (1995).

[13] A.P. Pyatakov et.al., MSU Phys. Bull. 65, 329-331 (2010). 